\title{
Individualized Dosimetry for Theranostics: Necessary, Nice to Have, or Counterproductive?
}

\author{
Uta Eberlein $^{1}$, Marta Cremonesi ${ }^{2}$, and Michael Lassmann ${ }^{1}$ \\ ${ }^{I}$ Klinik und Poliklinik für Nuklearmedizin, Universitätsklinikum Würzburg, Würzburg, Germany; and ${ }^{2}$ Radiation Research Unit, \\ Istituto Europeo di Oncologia, Milano, Italy
}

\begin{abstract}
In 2005, the term theragnostics (theranostics) was introduced for describing the use of imaging for therapy planning in radiation oncology. In nuclear medicine, this expression describes the use of tracers for predicting the absorbed doses in molecular radiotherapy and, thus, the safety and efficacy of a treatment. At present, the most successful groups of isotopes for this purpose are ${ }^{123}\left|/{ }^{124}\right| /{ }^{131} \mid,{ }^{68} \mathrm{Ga} /{ }^{177} \mathrm{Lu}$, and ${ }^{111} \mathrm{In} /{ }^{86} \mathrm{Y} /{ }^{90} \mathrm{Y}$. The purpose of this review is to summarize available data on the dosimetry and dose-response relationships of several theranostic compounds, with a special focus on radioiodine therapy for differentiated thyroid cancer and peptide receptor radionuclide therapy. These are treatment modalities for which dose-response relationships for healthy tissues and tumors have been demonstrated. In addition, available data demonstrate that posttherapeutic dosimetry after a first treatment cycle predicts the absorbed doses in further cycles. Both examples show the applicability of the concept of theranostics in molecular radiotherapies. Nevertheless, unanswered questions need to be addressed in clinical trials incorporating dosimetry-related concepts for determining the amount of therapeutic activity to be administered.
\end{abstract}

Key Words: absorbed dose; dosimetry; radiobiology; radioiodine therapy; PRRT; theranostics

J Nucl Med 2017; 58:97S-103S

DOI: 10.2967/jnumed.116.186841

$\mathbf{I}$

n 2005, the term theragnostics (theranostics) was first used for describing the use of imaging for therapy planning in radiation oncology. It is defined as the use of information from medical images to determine the optimal therapy for an individual patient (1). In nuclear medicine, this expression describes the use of tracers for predicting the absorbed doses in molecular radiotherapy and, thus, the safety and efficacy of a treatment. At present, the most successful groups of isotopes for theranostics are ${ }^{123} \mathrm{I} /{ }^{124} \mathrm{I} /{ }^{131} \mathrm{I}(2),{ }^{68} \mathrm{Ga} /{ }^{177} \mathrm{Lu}$, and ${ }^{111} \mathrm{In} /{ }^{86} \mathrm{Y} /{ }^{90} \mathrm{Y}(3)$.

In recent years, numerous reports providing pre- and peritherapeutic dosimetry data for molecular radiotherapies with ${ }^{131}$ I-sodium iodide (4-8) and ${ }^{177} \mathrm{Lu}$-labeled (9-16) and ${ }^{90}$ Y-labeled (13,17-20) compounds have been published. The purpose of this review is to introduce the concept of theranostics, present available data on the dosimetry and dose-response relationships of theranostic com-

Received Jan. 20, 2017; revision accepted Mar. 10, 2017.

For correspondence or reprints contact: Michael Lassmann, Department of Nuclear Medicine, University of Würzburg, Oberdürrbacher Straße 6, 97080 Würzburg, Germany.

E-mail: Lassmann_m@ukw.de

COPYRIGHT @ 2017 by the Society of Nuclear Medicine and Molecular Imaging. pounds, and discuss whether individualized dosimetry for theranostics is necessary, nice to have, or counterproductive. Special focus is given to the provision of radioiodine therapy for differentiated thyroid cancer and peptide receptor radionuclide therapy (PRRT) with ${ }^{177} \mathrm{Lu}-$ and ${ }^{90}$ Y-labeled peptides.

\section{INTERNAL DOSIMETRY}

\section{Dosimetry in Nuclear Medicine}

The basic methodology for calculating the absorbed doses from the administration of a radiopharmaceutical was developed by the MIRD Committee (21):

$$
D\left(r_{T}\right)=\tilde{A}\left(r_{S}\right) \cdot S\left(r_{T} \leftarrow r_{S}\right)=A_{0} \cdot \tilde{a}\left(r_{S}\right) \cdot S\left(r_{T} \leftarrow r_{S}\right),
$$

Eq. 1

where

- $D\left(r_{T}\right)$ is the mean absorbed dose to target region $r_{T}$ delivered by the cumulated activity in source region $r_{S}$.

- $\tilde{A}\left(r_{S}\right)$ is the time-integrated activity in source region $r_{S} . \tilde{A}$ denotes the total number of radioactive decays (determined by integrating the time-activity curve from time 0 to infinity) occurring within an accumulating organ. $\tilde{A}$ is determined by quantitative imaging at several time points after the administration of a radiopharmaceutical for an assessment of organor voxel-specific pharmacokinetics. A proposal for choosing optimal time points is given in MIRD Pamphlet No. 16 (22).

- $A_{O}$ is the administered activity.

- $\tilde{\mathrm{a}}\left(r_{S}\right)$ is the time-integrated activity coefficient, which is the time-integrated activity divided by the administered activity.

- $S\left(r_{T} \leftarrow r_{S}\right)$ is the radionuclide specific absorbed dose rate per unit of activity in target region $r_{T}$ delivered by source region $r_{S}$ (formerly called $\mathrm{S}$ value).

For calculation of the absorbed dose, therefore, several steps are essential.

The first step is to perform quantitative imaging at different time points to assess the activity in the organs of interest over time. The established method for quantitative imaging for dosimetry relies on the measurement of biokinetics by serial $\gamma$-camera scans. The use of SPECT/CT (or, if applicable, PET/CT) increases quantitative accuracy to a large extent, as shown by Zimmerman et al. in a multicenter trial (23). Quantitative accuracy depends on reliable calibration and careful adjustment of the camera and reconstruction settings. For organ or lesion dosimetry, individual organ or lesion volumes must be determined; for example, by CT the use of standard organ volumes may be incorrect and may lead to serious under- or overestimation of the absorbed doses.

The second step is to generate the time-activity curve for accumulating organs and lesions and to integrate this curve over time 
to obtain the total number of decays occurring in the source organs. For this purpose, it is essential to select the optimal time points and the optimal fit function. A proposal for choosing the optimal time points is provided in MIRD Pamphlet No. 16 (22), and a proposal for obtaining optimal fit procedures for nuclear medicine is provided by Kletting et al. (24).

The last step is the choice of the $S$ value. The $S$ value accounts for the energy released from each radioactive decay and the relative geometry of the source organ and the organ for which the absorbed dose is to be calculated. Thus, the cumulated activity is dependent on biologic parameters, whereas the $\mathrm{S}$ factor takes into account the physical components of the absorbed dose. No assumption concerning the source or target is made, other than that the distribution of the activity is homogeneous in source $r_{S}$. The source and the target can be of any size or composition. Theoretically, if the activity in the source is heterogeneously distributed, then it is possible to subdivide the source into smaller volumes in which the activity can be considered homogeneous.

$S$ value calculations strongly depend on the choice of the model. If tabulated $S$ values calculated from mathematic anthropomorphic phantoms are used, then the masses of the organs should be adjusted to the weights of the patients' organs. At present, voxelbased $\mathrm{S}$ values are frequently used for patient-specific dose calculations. An example of the type of calculation that uses voxel-based $\mathrm{S}$ values is the NUKDOS software (25).

\section{Radiobiology in Nuclear Medicine}

During internal irradiation in nuclear medicine-in contrast to external irradiation - the cells and organs are continuously irradiated over a longer period with a permanently changing dose rate. This might modify the impact of the ionizing radiation although the same absorbed dose is delivered. Because of the continuous irradiation in nuclear medicine, the repair of cell damage is already taking place during therapy (26).

For comparison of therapies with different dose rates and fractionation schemes, the concept of the biologically effective dose (BED) has been introduced (27-29):

$$
B E D\left(D\left(r_{T}\right)\right)=D\left(r_{T}\right) \cdot R E\left(D\left(r_{T}\right)\right),
$$

where $\operatorname{BED}\left(D\left(r_{T}\right)\right)$ is the biologically effective dose for the absorbed dose to the target region $r_{T}, D\left(r_{T}\right)$ defines the absorbed dose to the target region, and $\operatorname{RE}\left(D\left(r_{T}\right)\right)$ defines the relative effectiveness; the latter must be modified for nuclear medicine by factor $G$, which takes into account the repair of sublethal damage taking place during therapy:

$$
R E\left(D\left(r_{T}\right)\right)=1+\cdot \frac{G}{\alpha / \beta} \cdot D\left(r_{T}\right)
$$

Assuming a monoexponential decrease in dose rate, $G$ can be written as:

$$
G=\frac{\lambda}{\lambda+\mu}
$$

with

$$
\lambda=\frac{\ln (2)}{t_{1 / 2}},
$$

where $\lambda$ is the effective rate of decay of the nuclide from the organ of interest, $t_{1 / 2}$ is the effective half-life, and $\mu$ is the sub- lethal damage recovery constant (assuming an exponential repair rate).

$\alpha$ and $\beta$ are tissue-specific constants that represent the survival curves of cells. Cell survival after exposure to ionizing radiation is usually represented by the linear quadratic model (29):

$$
F_{S}(D)=\exp \left(-\alpha \cdot D-\beta \cdot D^{2}\right)
$$

where $F_{S}$ is the cell survival fraction, $D$ is the absorbed dose, $\alpha$ describes lethal cell damage for which only a single event is necessary, and $\beta$ describes lethal events that require 2 sublethal events (28). Using Equations 2 and 3, the survival fraction can then be rewritten as:

$$
\begin{aligned}
F_{S}(D) & =\exp \left(-\alpha \cdot D \cdot\left(1+\frac{G}{\alpha / \beta} \cdot D\right)\right) . \\
& =\exp (-\alpha \cdot B E D(D))
\end{aligned}
$$

The $\alpha / \beta$ ratio describes the absorbed dose for which the cell killing represented by the linear and quadratic parts of Equation 6 are equal.

For more than 1 therapy cycle, the BEDs can be summed, given the assumptions that there is no activity left and that the sublethal damage is repaired between the cycles (26).

How this concept can be applied to kidney dosimetry in PRRT, for instance, with ${ }^{90} \mathrm{Y}$ - and ${ }^{177} \mathrm{Lu}$-labeled compounds, is illustrated in Figure 1. Data for the $\alpha / \beta$ ratio, the repair half-life, and the effective half-life were taken from MIRD Pamphlet No. 20 (29). Bodei et al. (30) observed a threshold for kidney toxicity at a BED of $40 \mathrm{~Gy}$ in patients without risk factors. In Figure 1, the dotted lines denote the intersection of a BED of $40 \mathrm{~Gy}$ and the corresponding absorbed doses to the kidney for 1 and 4 therapy cycles with ${ }^{90} \mathrm{Y}$ ( 25 and $33 \mathrm{~Gy}$ ) and ${ }^{177} \mathrm{Lu}(28$ and $35 \mathrm{~Gy}$ ). This graph also shows that the normally accepted tolerable dose for healthy kidneys (23 Gy (31)) could be exceeded if the therapy were fractionated and the BED formalism were applied.

Specific kidney dosimetry-based randomized clinical trials have not been conducted yet. There is, however, evidence that fractionation allows renal sparing in ${ }^{90}$ Y-PRRT. Absorbed doses

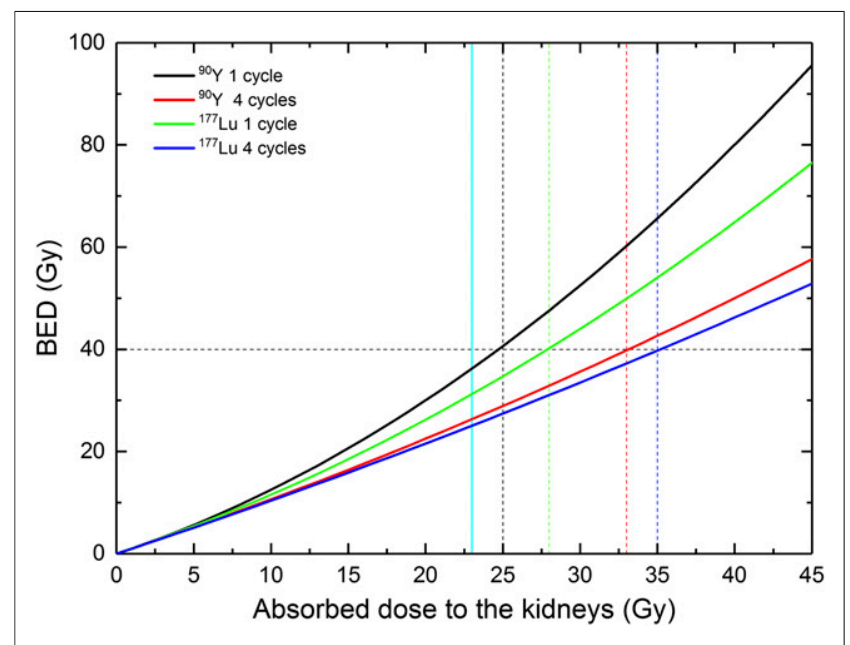

FIGURE 1. BED as function of absorbed dose for healthy kidneys after PRRT with ${ }^{90} \mathrm{Y}$ - and ${ }^{177} \mathrm{Lu}$-labeled compounds. Dotted lines denote intersection of BED of 40 Gy and corresponding cycle- and nuclide-dependent absorbed kidney doses. Vertical light blue solid line denotes normally accepted tolerable absorbed dose (23 Gy) for healthy kidneys. 
to the kidneys were generally higher with this treatment regimen than with ${ }^{177} \mathrm{Lu}$-PRRT protocols due to the fixed activities administered in the corresponding clinical trials $(30,32-34)$. In particular, an incidence of grade IV permanent renal toxicity of higher than $9 \%$ was observed after the administration of activities as high as $3.7 \mathrm{GBq} / \mathrm{m}^{2}$ of body surface area (corresponding to $\sim 6.3 \mathrm{GBq}$ ) per cycle in 1-10 cycles per patient (34), whereas a much lower frequency-or just grade 1 toxicity-was observed by other authors who used more prudent approaches $(35,36)$ involving lower activity and more cycles (19).

\section{APPLICATIONS}

\section{Radioiodine Therapy of Differentiated Thyroid Cancer}

Although ${ }^{123} \mathrm{I}$ is suited for detecting metastases in thyroid cancer patients with conventional SPECT/CT systems, the usefulness of this short-lived isotope for a theranostics concept in thyroid cancer has not been shown (2). The isotope ${ }^{124} \mathrm{I}$ is preferable for theranostics because it has 2 main advantages: the better resolution of PET than of SPECT and the longer half-life, which allows the collection of quantitative data for days after administration.
Several institutions have demonstrated the use of ${ }^{124} \mathrm{I}$ as a substitute for ${ }^{131}$ I for pretherapeutic dosimetry. Sgouros et al. (37) assessed spatial distributions of absorbed doses, isodose contours, dose-volume histograms, and mean absorbed dose estimates for a total of 56 tumors in 15 patients. The mean absorbed doses for individual tumors ranged from 1.2 to $540 \mathrm{~Gy}$. Sgouros et al. demonstrated that ${ }^{124}$ I PET-based, patient-specific 3-dimensional dosimetry is feasible and that sequential PET can be used to obtain time-integrated activity coefficients for 3-dimensional dosimetry.

In a recently published report on pretherapeutic dosimetry using PET/CT with ${ }^{124}$ I, Wierts et al. (38) confirmed the results of Maxon et al. (39) regarding the absorbed doses needed for ablation $(>300$ Gy) and of Maxon and Smith (40) regarding the successful treatment of metastases ( $>80 \mathrm{~Gy})$.

All in all, despite the limited availability of ${ }^{124} \mathrm{I}$, these reports show the successful application of a theranostics concept to diagnostics and therapy in differentiated thyroid cancer.

\section{PRRT with ${ }^{177} \mathrm{Lu}$}

For ${ }^{177} \mathrm{Lu}$-labeled peptides, Table 1 summarizes data on absorbed doses for the red marrow, kidneys, and tumors from various studies.

\section{TABLE 1}

Absorbed Doses for Tumors and Organs at Risk in ${ }^{177}$ Lu PRRT Studies

\begin{tabular}{|c|c|c|c|c|c|c|}
\hline \multirow[b]{2}{*}{ Organ or lesion } & \multirow[b]{2}{*}{ No. of patients } & \multicolumn{3}{|c|}{ Absorbed dose (Gy/GBq) } & \multirow[b]{2}{*}{ Method } & \multirow[b]{2}{*}{ Reference } \\
\hline & & Median & Range & Mean \pm SD & & \\
\hline \multirow[t]{7}{*}{ Red marrow } & 6 & & & $0.07 \pm 0.01$ & Blood & 9 \\
\hline & 61 & & & $0.04 \pm 0.02$ & Blood & 10 \\
\hline & 15 & 0.02 & $0.01-0.13$ & $0.034 \pm 0.030$ & Blood & 11 \\
\hline & 12 & 0.03 & $0.02-0.06$ & $0.04 \pm 0.02$ & Blood & 13 \\
\hline & 200 & 0.02 & $0.01-0.05$ & & Blood & 14 \\
\hline & 7 & $\leq 0.07^{\star}\left(\leq 0.08^{\dagger}\right)$ & & & SPECT & 16 \\
\hline & 10 & 0.04 & $0.02-0.06$ & & Blood & 33 \\
\hline \multirow[t]{10}{*}{ Kidneys } & 6 & & & $0.88 \pm 0.19$ & Planar & 9 \\
\hline & 61 & & & $0.90 \pm 0.30$ & Planar & 10 \\
\hline & 16 & & & $0.97 \pm 0.24$ & Planar & 12 \\
\hline & 16 & & & $0.90 \pm 0.21$ & SPECT & 12 \\
\hline & 12 & 0.68 & $0.33-1.65$ & $0.80 \pm 0.35$ & Planar & 13 \\
\hline & 200 & 0.61 & $0.27-1.35$ & & SPECT & 14 \\
\hline & 88 & & $0.36-0.78$ & $0.57 \pm 0.09$ & Planar & 15 \\
\hline & 7 & $1.15^{\star}\left(0.68^{\dagger}\right)$ & $0.54-2.16^{*}\left(0.34-1.82^{\dagger}\right)$ & $1.24 \pm 0.49^{*}\left(0.84 \pm 0.49^{\dagger}\right)$ & SPECT & 16 \\
\hline & 10 & 0.62 & $0.45-17.74$ & & Planar & 33 \\
\hline & 33 & & $0.33-2.4$ & $0.8 \pm 0.3$ & Planar & 42 \\
\hline \multirow[t]{7}{*}{ Tumors } & 6 & & $3.9-37.9$ & & Planar & 9 \\
\hline & 61 & & & $9.7 \pm 11.1$ & Planar & 10 \\
\hline & 16 & 6.7 & $0.1-20$ & & SPECT & 12 \\
\hline & 88 & & $1.3-4.8$ & $3.41 \pm 0.68$ & Planar & 15 \\
\hline & 7 & & $2-11^{*}\left(1-11^{\dagger}\right)$ & & SPECT & 16 \\
\hline & 10 & & $0.6-56$ & & Planar & 33 \\
\hline & 24 & 6.8 & $1.4-23$ & & SPECT & 42 \\
\hline
\end{tabular}

${ }^{*}$ Pretherapeutic.

†Posttherapeutic. 
The values were all obtained from one of the therapeutic cycles in ${ }^{177} \mathrm{Lu}$ dosimetry studies.

Concerning red marrow absorbed doses and hematologic toxicity, the ${ }^{177} \mathrm{Lu}$-labeled compounds showed rapid clearance from the blood (14). The absorbed doses calculated using bloodbased methods resulted in low specific absorbed doses-less than $0.13 \mathrm{~Gy} / \mathrm{GBq}$. Consequently, high-grade hematologic toxicity has not been observed in patients receiving ${ }^{177} \mathrm{Lu}$-DOTATATE or ${ }^{177}$ Lu-DOTATOC; mostly grade 3 leukopenia and thrombocytopenia have been noted $(13,32)$. An interesting observation was reported by Forrer et al. (11). They observed a strong linear correlation and a high level of agreement between the measured radioactivity in bone marrow aspirates and that in the blood. These data should sustain the use of a blood-based model for red marrow absorbed dose estimates, at least for ${ }^{177} \mathrm{Lu}$-PRRT. Unfortunately, a correlation between absorbed dose and toxicity could not be derived.

The mean absorbed doses to the kidney varied from 0.57 to 0.97 Gy/GBq; the variability was most likely caused by methodologic issues. In any case, variability among patients was intrinsically large because of patient-specific metabolism and the different forms of renal protection used.

In 1 study, differences between pretherapeutic dosimetry using $200 \mathrm{MBq}$ of ${ }^{177} \mathrm{Lu}$-DOTATAE and posttherapeutic dosimetry were observed. Although similar conditions were applied for kidney protection before diagnostic imaging and therapy, the pretherapeutic absorbed dose estimates were higher by almost a factor of 2 (16). However, the authors did not discuss potential reasons for these differences.
Other authors repeated renal dosimetry at each PRRT cycle and found minimal differences between cycles $(12,41)$.

In therapies with ${ }^{177} \mathrm{Lu}$-labeled peptides, acute or delayed renal toxicity has been observed only rarely (32). Therefore, the maximum tolerable absorbed dose of ${ }^{177} \mathrm{Lu}$ remains unknown, and these therapies are routinely performed without dosimetry in most cases.

Regarding tumor dosimetry, absorbed dose ranges are provided in Table 1. The wide inter- and intrapatient variability lowered the significance of the mean and median values (0.1-56 Gy/GBq) (Table 1). A particularly important result was obtained by Ilan et al. (42), who found a correlation between absorbed doses and tumor reduction (with correlation coefficients of 0.6 and 0.9 for tumors larger than 2.2 and $4 \mathrm{~cm}$, respectively).

\section{PRRT with ${ }^{90} \mathbf{Y}$}

Data on absorbed doses after therapy with ${ }^{90}$ Y-labeled compounds are sparse and are based mostly on pretherapeutic quantitative imaging using either ${ }^{111} \mathrm{In}$ - or ${ }^{86} \mathrm{Y}$-labeled peptides $(18,33,43-47)$.

The choice of ${ }^{111} \mathrm{In}$ or ${ }^{86} \mathrm{Y}$ for theranostics involves consideration of the advantages and drawbacks of both radionuclides, among which are the lower resolution with ${ }^{111}$ In and the insufficient time interval for data collection $(\sim 1 \mathrm{~d})$ with ${ }^{86} \mathrm{Y}$ because of its physical half-life $(14.7 \mathrm{~h})$; the latter represents a limit for estimation of the absorbed dose to the kidney $(33,48)$. In any case, the use of ${ }^{111} \mathrm{In}$ and ${ }^{86} \mathrm{Y}$ is supported by the fact that extrapolation from both provides reasonably similar estimates for ${ }^{90} \mathrm{Y}$.

With regard to the variability of absorbed doses, the same issues considered for PRRT with ${ }^{177} \mathrm{Lu}$ apply to PRRT with ${ }^{90} \mathrm{Y}$. Only a few studies on the absorbed doses to the red bone marrow have

TABLE 2

Absorbed Doses for ${ }^{90}$ Y, Renal Protection, ${ }^{*}$ Radionuclide Used for Theranostics, and Method Used for Dosimetry

\begin{tabular}{|c|c|c|c|c|c|c|c|c|}
\hline \multirow[b]{2}{*}{ Organ or lesion } & \multirow[b]{2}{*}{ No. of patients } & \multirow{2}{*}{$\begin{array}{c}\text { Renal } \\
\text { protection }\end{array}$} & \multicolumn{3}{|c|}{ Absorbed dose (Gy/GBq) } & \multirow{2}{*}{$\begin{array}{l}\text { Radionuclide for } \\
\text { theranostics }\end{array}$} & \multirow[b]{2}{*}{ Method } & \multirow[b]{2}{*}{ Reference } \\
\hline & & & Median & Range & Mean \pm SD & & & \\
\hline \multirow[t]{3}{*}{ Red marrow } & 18 & No & & & $0.03 \pm 0.01$ & ${ }^{111}$ In & Blood & 20 \\
\hline & 5 & No & 0.17 & $0.15-0.19$ & $0.17 \pm 0.02$ & ${ }^{111}$ In & Blood & 43 \\
\hline & 8 & Yes & & & $0.06 \pm 0.02$ & ${ }^{86} Y$ & Blood & 47 \\
\hline \multirow[t]{7}{*}{ Kidney } & 18 & No & & & $3.3 \pm 2.2$ & ${ }^{111}$ In & Planar & 20 \\
\hline & 5 & No & 3.0 & $1.9-3.6$ & $2.8 \pm 0.6$ & ${ }^{111}$ In & Planar & 43 \\
\hline & 4 & No & 4.8 & $3.1-5.2$ & $4.4 \pm 1.0$ & ${ }^{86} Y$ & PET & 44 \\
\hline & 6 & Yes & 1.8 & $1.4-2.0$ & $1.7 \pm 0.3$ & & & \\
\hline & 17 & Yes & 3.8 & $1.9-7.6$ & & ${ }^{111}$ In & SPECT & 45 \\
\hline & 8 & Yes & & $0.6-2.8$ & $1.7 \pm 0.9$ & ${ }^{86} Y$ & PET & 47 \\
\hline & 8 & No & & & 6.1 & 111 In & Planar & 56 \\
\hline \multirow[t]{7}{*}{ Tumor } & 18 & No & 9.6 & $1.4-31$ & $10.1 \pm 7.5$ & ${ }^{111}$ In & Planar & 20 \\
\hline & 5 & No & 12.2 & $2.4-41.7$ & $16.0 \pm 14.6$ & ${ }^{111}$ In & Planar & 43 \\
\hline & 4 & No & & & $2.0 \pm 0.7$ & ${ }^{86} Y$ & PET & 44 \\
\hline & 6 & Yes & & & $2.1 \pm 0.7$ & & & \\
\hline & $24^{\dagger}$ & & & $0.4-16.5$ & & & & \\
\hline & 17 & Yes & 3.8 & $0.9-5.4$ & & ${ }^{111} / \mathrm{n}$ & SPECT & 45 \\
\hline & 8 & Yes & & $2.1-29.5$ & $9.0 \pm 6.6$ & ${ }^{86} Y$ & PET & 47 \\
\hline
\end{tabular}

*Many data precede studies on renal protection.

${ }^{\dagger}$ Renal protection was not specified. 
been done; the mean absorbed doses varied from 0.03 to $0.17 \mathrm{~Gy} /$ GBq (Table 2).

The mean absorbed doses to the kidneys varied from 1.7 to 6.1 Gy/GBq (Table 2). The variability was most likely caused by methodologic issues (higher values in ${ }^{111}$ In scans than in ${ }^{86} \mathrm{Y}$ PET scans). Although data are available on toxicity thresholds (typically a BED of 40 Gy (29)), therapy with ${ }^{90}$ Y-labeled peptides is routinely performed without dosimetry in most cases. The absorbed doses to the tumors showed a wide variability, ranging from 0.4 to $41.7 \mathrm{~Gy} / \mathrm{GBq}$.

In a recent study, Strigari et al. (49) analyzed radionuclide therapy-related dose-effect relationships. For neuroendocrine tumors, their review concentrated on absorbed-dose effects concerning bone marrow and kidney toxicity. The corresponding studies are summarized in Table 3.

In a patient-specific dosimetry study with the aim of predicting renal toxicity with ${ }^{90}$ Y-DOTATOC, Barone et al. found a therapyrelated dose-effect relationship for renal failure (18). The endpoint was a reduction in creatinine clearance of greater than $20 \%$ per year, as not all patients developed G3 or G4 nephrotoxicity. This value was obtained only when individual kidney masses were considered and the BED ( $>40 \mathrm{~Gy}$ ) was used as a dosimetric descriptor, accounting for different dosing schemes $(18,29)$. Bodei et al. (30) observed 2 dose limits for kidneys after treatment with ${ }^{90} \mathrm{Y}$ : a BED of 28 Gy was a threshold for toxicity in patients with risk factors (mainly hypertension and diabetes), and a BED of $40 \mathrm{~Gy}$ was the limit in patients without risk factors.

These findings were confirmed by a prospective phase 2 dosimetry trial in which a threshold of BED of 37 Gy for kidney toxicity was found to be a good guide for ${ }^{90}$ Y-DOTATOC PRRT (50).

With respect to hematologic toxicity in 21 patients with neuroendocrine tumors treated with ${ }^{90}$ Y-DOTATOC and showing a recovery of platelet counts to normal, Walrand et al. (51) found a strong correlation between the absorbed doses to the red marrow and the platelet count reduction at the nadir in 9 patients who were selected a posteriori, who had not had chemotherapy, and who had spontaneous red marrow recovery. A 50\% reduction in the platelet counts was observed at an absorbed dose to the red marrow of $2 \mathrm{~Gy}$ (51). However, the authors reported only the absorbed doses and did not provide information about the administered ${ }^{90} \mathrm{Y}$ activity; therefore, the specific absorbed doses could not be reported in Table 3 .

\section{Can Diagnostic Scans with ${ }^{68} \mathrm{Ga}$ Predict Posttherapeutic Absorbed Doses?}

In principle, dosimetry with ${ }^{68} \mathrm{Ga}$-labeled peptides cannot be directly extrapolated for PRRT-especially for normal organsbecause the half-life of ${ }^{68} \mathrm{Ga}$ is too short compared with the effective half-lives of ${ }^{177} \mathrm{Lu}$ - and ${ }^{90}$ Y-labeled peptides $(>30 \mathrm{~h})$. Moreover, because normal organs (typically liver and spleen but sometimes also kidneys) and tumors may show an uptake phase for more than $24 \mathrm{~h}$, accurate biokinetics and related areas under the time-activity curves cannot be assessed appropriately (48).

Nevertheless, 2 retrospective studies demonstrated correspondence between early lesion uptake and absorbed dose after PET scans with ${ }^{68} \mathrm{Ga}$-labeled somatostatin analogs, allowing the pretherapeutic assessment of tumor radionuclide uptake in PRRT in a relatively small number of patients $(52,53)$.

Hänscheid et al. (52) investigated whether the tumor uptake of a radionuclide in PRRT of meningioma could be predicted by a PET scan with ${ }^{68} \mathrm{Ga}$-labeled somatostatin analogs in 11 patients. They concluded that PET with ${ }^{68} \mathrm{Ga}$-labeled somatostatin analogs allows the pretherapeutic assessment of tumor radionuclide uptake in PRRT of meningioma and an estimate of the achievable dose.

In another study, Ezziddin et al. (53) compared SUVs and absorbed doses in 21 patients who had 61 evaluable tumor lesions and were undergoing both pretherapeutic ${ }^{68} \mathrm{Ga}$-DOTATOC PET/ CT and PRRT with ${ }^{177} \mathrm{Lu}$-octreotate. They found that somatostatin receptor PET imaging may predict tumor absorbed doses. The indication of insufficient target irradiation by a low SUV could aid in the selection of appropriate candidates for PRRT.

\section{INDIVIDUALIZED DOSIMETRY: NECESSARY, NICE TO HAVE, OR COUNTERPRODUCTIVE?}

Several studies have demonstrated that a theranostic concept can be applied to predicting absorbed doses. The most successful example is the pair ${ }^{124} \mathrm{I} /{ }^{131} \mathrm{I}$; further studies are needed to show the applicability of the short-lived PET tracer ${ }^{68} \mathrm{Ga}$ for predicting therapeutic absorbed doses.

For PRRT with ${ }^{90} \mathrm{Y}$, pretherapeutic dosimetry data based on calculations of the absorbed doses and the BED demonstrated a clear relationship between the BED and kidney toxicity at a dose limit of a BED of $40 \mathrm{~Gy}$. However, dosimetry after the administration of ${ }^{90} \mathrm{Y}$ remains problematic because of inherent difficulties with quantitative bremsstrahlung imaging with $\gamma$-cameras.

Peritherapeutic dosimetry in molecular radiotherapies with ${ }^{177} \mathrm{Lu}$-labeled compounds, although sometimes limited by the availability of patients for late-time-point measurements, can be easily performed with limited technical difficulties (54). Most importantly, the demonstration of a tumor dose-response relationship (42) and the observation of minimal differences between cycles $(12,41)$ indicate that posttherapeutic dosimetry after a first treatment cycle predicts the absorbed doses in further cycles.

The following questions should be addressed by dosimetry for therapies with ${ }^{177} \mathrm{Lu}$-labeled compounds: Can imaging with shortlived PET nuclides predict the toxicity or efficacy of a treatment? What is the limit of the absorbed doses to the kidneys, as such a

TABLE 3

Dose-Effect Relationships for Kidneys and Bone Marrow After PRRT with ${ }^{90} \mathrm{Y}$

\begin{tabular}{lll}
\hline \multicolumn{1}{c}{ Endpoint } & \multicolumn{1}{c}{ Threshold } & Reference \\
\hline$>20 \%$ decline in creatinine clearance/y & A BED of 45 Gy (kidneys) & 18 \\
$50 \%$ reduction in platelets & Absorbed dose of 2 Gy (bone marrow) & 51 \\
Creatinine toxicity exceeding grade 1 & $\begin{array}{l}\text { A BED of 28 Gy in presence of kidney risk } \\
\text { factors; a BED of 40 Gy in absence of } \\
\text { kidney risk factors }\end{array}$ \\
\hline
\end{tabular}


limit based on patient data has not been established yet for peptide therapies with ${ }^{177} \mathrm{Lu}$-labeled compounds? What is the optimal number of therapy cycles? What are the influences of fractionation of therapies? What is the role of pharmacokinetic modeling in predicting the effects of cold peptides or small molecules? How can existing methods for bone marrow dosimetry be amended to predict acute hematologic toxicities?

In addition, as shown by Pfestroff et al. (55) for prostate-specific membrane antigen-targeted therapy, a harmonization of therapy protocols and the establishment of standardized dosimetry protocols for assessing safety and efficacy are urgently needed.

There are still gray zones in the clinical applications of dosimetry. One of them is the lack of standardization for quantitative imaging using SPECT/CT across centers. To address this problem, efforts should be undertaken to create an accreditation program similar to that for ${ }^{18} \mathrm{~F}$-FDG PET. Further research is needed to optimize the timing and the number of time points for patient scans, the integration of the time-activity curves, and the development of patient-specific $S$ values. Contributions to the questions listed earlier can be expected from the results of the European research project MRTDOSIMETRY ("Metrology for Clinical Implementation of Dosimetry in Molecular Radiotherapy"; http://mrtdosimetry-empir.eu).

Another topic that should be addressed by dosimetry is the determination of kidney dose limits. For many clinical trials, a maximum absorbed dose of $23 \mathrm{~Gy}$ has been adopted on the basis of data from external-beam therapy. Dosimetry-based kidney dose escalation trials are required, particularly for therapies involving ${ }^{177} \mathrm{Lu}$, to establish dose limits for patient-tailored treatment.

\section{CONCLUSION}

Dosimetry not only is nice to have and easily performed after treatment with ${ }^{177} \mathrm{Lu}$ or before treatment of differentiated thyroid cancer with radioiodine but also is needed for predicting therapy success and optimizing therapeutic applications of radiopharmaceuticals. The disadvantage of an increased need for resources is easily counterbalanced by the patient-specific information that is provided by dosimetry for optimizing the treatment of each patient.

\section{DISCLOSURE}

No potential conflict of interest relevant to this article was reported.

\section{REFERENCES}

1. Bentzen SM. Theragnostic imaging for radiation oncology: dose-painting by numbers. Lancet Oncol. 2005;6:112-117.

2. Luster M, Clarke SE, Dietlein M, et al. Guidelines for radioiodine therapy of differentiated thyroid cancer. Eur J Nucl Med Mol Imaging. 2008;35:1941-1959.

3. Bodei L, Mueller-Brand J, Baum RP, et al. The joint IAEA, EANM, and SNMMI practical guidance on peptide receptor radionuclide therapy (PRRNT) in neuroendocrine tumours. Eur J Nucl Med Mol Imaging. 2013;40:800-816.

4. Freudenberg LS, Jentzen W, Gorges R, et al. ${ }^{124}$ I-PET dosimetry in advanced differentiated thyroid cancer: therapeutic impact. Nuklearmedizin. 2007;46:121-128.

5. Jentzen W, Freudenberg L, Eising EG, Sonnenschein W, Knust J, Bockisch A. Optimized ${ }^{124}$ I PET dosimetry protocol for radioiodine therapy of differentiated thyroid cancer. J Nucl Med. 2008;49:1017-1023.

6. Jentzen W, Hobbs RF, Stahl A, Knust J, Sgouros G, Bockisch A. Pre-therapeutic ${ }^{124} \mathrm{I}$ PET(/CT) dosimetry confirms low average absorbed doses per administered ${ }^{131}$ I activity to the salivary glands in radioiodine therapy of differentiated thyroid cancer. Eur J Nucl Med Mol Imaging. 2010;37:884-895.
7. Jentzen W, Verschure F, van Zon A, et al. ${ }^{124} \mathrm{I}$ PET assessment of response of bone metastases to initial radioiodine treatment of differentiated thyroid cancer. J Nucl Med. 2016;57:1499-1504.

8. Ruhlmann M, Jentzen W, Ruhlmann V, et al. High level of agreement between pretherapeutic ${ }^{124} \mathrm{I}$ PET and intratherapeutic ${ }^{131} \mathrm{I}$ imaging in detecting iodinepositive thyroid cancer metastases. J Nucl Med. 2016;57:1339-1342.

9. Kwekkeboom DJ, Bakker WH, Kooij PP, et al. [177Lu-DOTAOTyr3]octreotate: comparison with [ ${ }^{111}$ In-DTPAo]octreotide in patients. Eur J Nucl Med. 2001;28: 1319-1325.

10. Wehrmann C, Senftleben S, Zachert C, Muller D, Baum RP. Results of individual patient dosimetry in peptide receptor radionuclide therapy with ${ }^{177} \mathrm{Lu}$ DOTATATE and ${ }^{177}$ Lu DOTA-NOC. Cancer Biother Radiopharm. 2007;22:406-416.

11. Forrer F, Krenning EP, Kooij PP, et al. Bone marrow dosimetry in peptide receptor radionuclide therapy with $\left[{ }^{177} \mathrm{Lu}^{-\mathrm{DOTA}_{0}}, \mathrm{Tyr}_{3}\right]$ octreotate. Eur J Nucl Med Mol Imaging. 2009;36:1138-1146.

12. Garkavij M, Nickel M, Sjögreen-Gleisner K, et al. ${ }^{177}$ Lu-[DOTA0,Tyr3] octreotate therapy in patients with disseminated neuroendocrine tumors: analysis of dosimetry with impact on future therapeutic strategy. Cancer. 2010;116(4 suppl): 1084-1092.

13. Bodei L, Cremonesi M, Grana CM, et al. Peptide receptor radionuclide therapy with ${ }^{177} \mathrm{Lu}$-DOTATATE: the IEO phase I-II study. Eur J Nucl Med Mol Imaging. 2011;38:2125-2135.

14. Sandström M, Garske-Roman U, Granberg D, et al. Individualized dosimetry of kidney and bone marrow in patients undergoing ${ }^{177} \mathrm{Lu}$-DOTA-octreotate treatment. J Nucl Med. 2013;54:33-41.

15. Gupta SK, Singla S, Thakral P, Bal CS. Dosimetric analyses of kidneys, liver, spleen, pituitary gland, and neuroendocrine tumors of patients treated with ${ }^{177}$ Lu-DOTATATE. Clin Nucl Med. 2013;38:188-194.

16. Kairemo K, Kangasmaki A. 4D SPECT/CT acquisition for 3D dose calculation and dose planning in ${ }^{177} \mathrm{Lu}$-peptide receptor radionuclide therapy: applications for clinical routine. Recent Results Cancer Res. 2013;194:537-550.

17. Paganelli G, Zoboli S, Cremonesi M, et al. Receptor-mediated radiotherapy with ${ }^{90}$ Y-DOTA-D-Phe1-Tyr3-octreotide. Eur J Nucl Med. 2001;28:426-434.

18. Barone R, Borson-Chazot F, Valkema R, et al. Patient-specific dosimetry in predicting renal toxicity with ${ }^{90} \mathrm{Y}-\mathrm{DOTATOC}$ : relevance of kidney volume and dose rate in finding a dose-effect relationship. J Nucl Med. 2005;46(suppl 1): 99S-106S.

19. Bodei L, Cremonesi M, Grana C, et al. Receptor radionuclide therapy with ${ }^{90} \mathrm{Y}$ [DOTA]0-Tyr3-octreotide ( ${ }^{90}$ Y-DOTATOC) in neuroendocrine tumours. Eur $J$ Nucl Med Mol Imaging. 2004;31:1038-1046.

20. Cremonesi M, Ferrari M, Zoboli S, et al. Biokinetics and dosimetry in patients administered with ${ }^{111} \mathrm{In}$-DOTA-Tyr 3 -octreotide: implications for internal radiotherapy with ${ }^{90}$ Y-DOTATOC. Eur J Nucl Med. 1999;26:877-886.

21. Bolch WE, Eckerman KF, Sgouros G, Thomas SR. A generalized schema for radiopharmaceutical dosimetry: standardization of nomenclature-MIRD Pamphlet No. 21. J Nucl Med. 2009;50:477-484.

22. Siegel JA, Thomas SR, Stubbs JB, et al. Techniques for quantitative radiopharmaceutical biodistribution data acquisition and analysis for use in human radiation dose estimates: MIRD Pamphlet No. 16. J Nucl Med. 1999;40:37S-61S.

23. Zimmerman BE, Grošev D, Buvat I, et al. Multi-centre evaluation of accuracy and reproducibility of planar and SPECT image quantification: an IAEA phantom study. Z Med Phys. April 19, 2016 [Epub ahead of print].

24. Kletting P, Schimmel S, Kestler HA, et al. Molecular radiotherapy: the NUKFIT software for calculating the time-integrated activity coefficient. Med Phys. 2013;40:102504.

25. Kletting P, Schimmel S, Haenscheid H, et al. NUKDOS: a software tool for dosimetry prior to molecular radiotherapy [abstract]. J Nucl Med. 2014;55(suppl 1): 1128 .

26. Eberlein U, Lassmann M. Internal dosimetry: principles and applications to NET. In: Pacak K, Taïeb D, eds. Diagnostic and Therapeutic Nuclear Medicine for Neuroendocrine Tumors. Cham, Switzerland: Springer International Publishing; 2017:201-217.

27. Dale R, Carabe-Fernandez A. The radiobiology of conventional radiotherapy and its application to radionuclide therapy. Cancer Biother Radiopharm. 2005;20:47-51.

28. Baechler S, Hobbs RF, Prideaux AR, Wahl RL, Sgouros G. Extension of the biological effective dose to the MIRD schema and possible implications in radionuclide therapy dosimetry. Med Phys. 2008;35:1123-1134.

29. Wessels BW, Konijnenberg MW, Dale RG, et al. The effect of model assumptions on kidney dosimetry and response: implications for radionuclide therapyMIRD Pamphlet No. 20. J Nucl Med. 2008;49:1884-1899.

30. Bodei L, Cremonesi M, Ferrari M, et al. Long-term evaluation of renal toxicity after peptide receptor radionuclide therapy with ${ }^{90}$ Y-DOTATOC and ${ }^{177} \mathrm{Lu}-\mathrm{DO}-$ TATATE: the role of associated risk factors. Eur J Nucl Med Mol Imaging. 2008;35:1847-1856. 
31. Emami B, Lyman J, Brown A, et al. Tolerance of normal tissue to therapeutic irradiation. Int J Radiat Oncol Biol Phys. 1991;21:109-122.

32. Kwekkeboom DJ, de Herder WW, Kam BL, et al. Treatment with the radiolabeled somatostatin analog $\left[{ }^{177} \mathrm{Lu}-\mathrm{DOTA}_{0}, \mathrm{Tyr}_{3}\right]$ octreotate: toxicity, efficacy, and survival. J Clin Oncol. 2008;26:2124-2130.

33. Cremonesi M, Botta F, Di Dia A, et al. Dosimetry for treatment with radiolabelled somatostatin analogues: a review. Q J Nucl Med Mol Imaging. 2010; 54:37-51.

34. Imhof A, Brunner P, Marincek N, et al. Response, survival, and long-term toxicity after therapy with the radiolabeled somatostatin analogue [ $\left.{ }^{90} \mathrm{Y}-\mathrm{DOTA}\right]-$ TOC in metastasized neuroendocrine cancers. J Clin Oncol. 2011;29:2416-2423.

35. Valkema R, Pauwels S, Kvols LK, et al. Survival and response after peptide

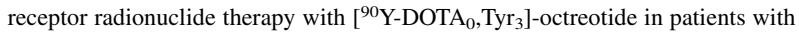
advanced gastroenteropancreatic neuroendocrine tumors. Semin Nucl Med. 2006;36:147-156

36. Bodei L, Cremonesi M, Zoboli S, et al. Receptor-mediated radionuclide therapy with ${ }^{90}$ Y-DOTATOC in association with amino acid infusion: a phase I study. Eur J Nucl Med Mol Imaging. 2003;30:207-216.

37. Sgouros G, Kolbert KS, Sheikh A, et al. Patient-specific dosimetry for ${ }^{131} \mathrm{I}$ thyroid cancer therapy using ${ }^{124} \mathrm{I}$ PET and 3-dimensional-internal dosimetry (3D-ID) software. J Nucl Med. 2004;45:1366-1372.

38. Wierts R, Brans B, Havekes B, et al. Dose-response relationship in differentiated thyroid cancer patients undergoing radioiodine treatment assessed by means of ${ }^{124}$ I PET/CT. J Nucl Med. 2016;57:1027-1032.

39. Maxon HR, Thomas SR, Hertzberg VS, et al. Relation between effective radiation dose and outcome of radioiodine therapy for thyroid cancer. $N$ Engl J Med. 1983;309:937-941.

40. Maxon HR III, Smith HS. Radioiodine-131 in the diagnosis and treatment of metastatic well differentiated thyroid cancer. Endocrinol Metab Clin North Am. 1990;19:685-718.

41. Larsson M, Bernhardt P, Svensson JB, Wängberg B, Ahlman H, ForssellAronsson E. Estimation of absorbed dose to the kidneys in patients after treatment with ${ }^{177} \mathrm{Lu}$-octreotate: comparison between methods based on planar scintigraphy. EJNMMI Res. 2012;2:49.

42. Ilan E, Sandstrom M, Wassberg C, et al. Dose response of pancreatic neuroendocrine tumors treated with peptide receptor radionuclide therapy using ${ }^{177} \mathrm{Lu}$ DOTATATE. J Nucl Med. 2015;56:177-182.

43. Forrer F, Uusijarvi H, Waldherr C, et al. A comparison of ${ }^{111}$ In-DOTATOC and ${ }^{111}$ In-DOTATATE: biodistribution and dosimetry in the same patients with metastatic neuroendocrine tumours. Eur J Nucl Med Mol Imaging. 2004;31:1257-1262.

44. Jamar F, Barone R, Mathieu I, et al. ${ }^{86} \mathrm{Y}_{-D O T A}-\mathrm{D}-\mathrm{Phe}_{1}-\mathrm{Tyr}_{3}$-octreotide (SMT487): a phase 1 clinical study-pharmacokinetics, biodistribution and renal protective effect of different regimens of amino acid co-infusion. Eur J Nucl Med Mol Imaging. 2003;30:510-518.

45. Hindorf C, Chittenden S, Causer L, Lewington VJ, Macke HR, Flux GD. Dosimetry for ${ }^{90}$ Y-DOTATOC therapies in patients with neuroendocrine tumors. Cancer Biother Radiopharm. 2007;22:130-135.

46. Förster GJ, Engelbach MJ, Brockmann JJ, et al. Preliminary data on biodistribution and dosimetry for therapy planning of somatostatin receptor positive tumours: comparison of ${ }^{86}$ Y-DOTATOC and ${ }^{111}$ In-DTPA-octreotide. Eur J Nucl Med. 2001;28:1743-1750.

47. Helisch A, Förster GJ, Reber H, et al. Pre-therapeutic dosimetry and biodistribution of ${ }^{86}$ Y-DOTA-Phe1-Tyr3-octreotide versus ${ }^{111}$ In-pentetreotide in patients with advanced neuroendocrine tumours. Eur J Nucl Med Mol Imaging. 2004;31: 1386-1392.

48. Guerriero F, Ferrari ME, Botta F, et al. Kidney dosimetry in ${ }^{177} \mathrm{Lu}$ and ${ }^{90} \mathrm{Y}$ peptide receptor radionuclide therapy: influence of image timing, time-activity integration method, and risk factors. Biomed Res Int. 2013;2013:935351.

49. Strigari L, Konijnenberg M, Chiesa C, et al. The evidence base for the use of internal dosimetry in the clinical practice of molecular radiotherapy. Eur J Nucl Med Mol Imaging. 2014;41:1976-1988.

50. Van Binnebeek S, Baete K, Vanbilloen B, et al. Individualized dosimetry-based activity reduction of ${ }^{90} \mathrm{Y}$-DOTATOC prevents severe and rapid kidney function deterioration from peptide receptor radionuclide therapy. Eur J Nucl Med Mol Imaging. 2014;41:1141-1157.

51. Walrand S, Barone R, Pauwels S, Jamar F. Experimental facts supporting a red marrow uptake due to radiometal transchelation in ${ }^{90}$ Y-DOTATOC therapy and relationship to the decrease of platelet counts. Eur J Nucl Med Mol Imaging. 2011;38:1270-1280.

52. Hänscheid H, Sweeney RA, Flentje M, et al. PET SUV correlates with radionuclide uptake in peptide receptor therapy in meningioma. Eur J Nucl Med Mol Imaging. 2012;39:1284-1288.

53. Ezziddin S, Lohmar J, Yong-Hing CJ, et al. Does the pretherapeutic tumor SUV in ${ }^{68} \mathrm{Ga}$ DOTATOC PET predict the absorbed dose of ${ }^{177} \mathrm{Lu}$ octreotate? Clin Nucl Med. 2012;37:e141-e147.

54. Ljungberg M, Celler A, Konijnenberg MW, et al. Joint EANM/MIRD guidelines for quantitative ${ }^{177} \mathrm{Lu}$ SPECT applied for dosimetry of radiopharmaceutical therapy: MIRD Pamphlet No. 26. J Nucl Med. 2016;57:151-162.

55. Pfestroff A, Luster M, Jilg CA, et al. Current status and future perspectives of PSMA-targeted therapy in Europe: opportunity knocks. Eur J Nucl Med Mol Imaging. 2015;42:1971-1975.

56. Kwekkeboom DJ, Kooij PP, Bakker WH, Macke HR, Krenning EP. Comparison of ${ }^{111}$ In-DOTA-Tyr ${ }^{3}$-octreotide and ${ }^{111}$ In-DTPA-octreotide in the same patients: biodistribution, kinetics, organ and tumor uptake. J Nucl Med. 1999;40:762-767. 